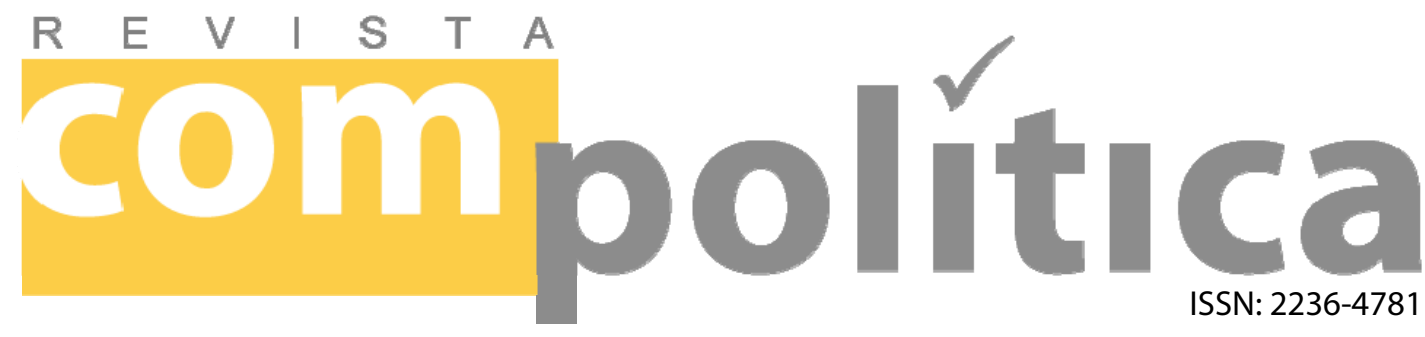

OTHON, Jambeiro

Phd, Professor Titular do ICI/UFBA

FERREIRA, Fábio

Phd, Professor Adjunto de ADM/UFBA

BARROS, Chalini

Doutoranda do PPGCOM/UFBA

\title{
A Convergência como condicionante da Regulação das Comunicações
}

\section{RESUMO}

fenômeno da convergência envolve uma combinação de transformações interligadas e interdependentes, de natureza tecnológica, industrial, comercial, cultural e social, que afetam a regulação das comunicações. Os usuários, por sua vez, tornaram-se igualmente convergentes, envolvidos numa cultura participativa intensa e, do ponto de vista do seu alcance geográfico e social, graças à convergência, cada vez mais extensa. Ao invés de consumidores passivos de mídia e serviços de informação e comunicação, temos agora utilizadores ativos e socialmente conectados, não mais silenciosos leitores/espectadores/ouvintes, mas ruidosos ativistas e publicistas. Compreender este fenômeno é essencial para vislumbrar um marco regulatório a ele apropriado. Este artigo procura definir convergência e discutir suas conseqüências, tendo no horizonte o provável debate que se espera que ocorra no Brasil, ainda este ano, sobre a revisão do marco regulatório das comunicações.

Palavras-chave: Regulação; Comunicação; Comunicações, Convergência Tecnológicas;

Políticas de Comunicação.

\section{ABSTRACT}

Convergence comprises a combination of interlinked and interdependent transformations, of technological, industrial, commercial, cultural and social nature, which affect communication regulation. Customers, at their time, turned also convergent, involved in an intense participative culture, which is too, from the point of view of its social and geographical range, thanks to convergence, more and more extense. Instead of passive consumers of media and information and communication services, we have now active and socially connected consumers, no more readers/spectators/listeners, but noisy activists and publishers. To understand this phenomenon is essential to discern a regulatory frame suitable for it. This Daper tries to define converaence and to discuss its conseavences. havina in the $h$ 


\title{
A Convergência como Condicionante da Regulação das Comunicações
}

JAMBEIRO, Othon, FERREIRA Fábio e BARROS, Chalini

\begin{abstract}
A
té meados da década de 1980 , cada serviço de comunicação conta disso, era com relativa facilidade que se podia desenhar instrumentos normativos difusos, de modo que os reguladores pudessem tratar cada indústria de comunicação sob um tipo de regime regulatório específico. Nos Estados Unidos, por exemplo, a manutenção (e regulação) da AT\&T como única provedora de serviços de telefonia justificava-se pela lógica dos monopólios naturais (alto custo de duplicação da rede). Além disso, uma vez que a indústria de telefonia baseava-se em conversações pessoa a pessoa, não fazia sentido haver regulação de conteúdo (tal regulação iria representar uma invasão injustificável de privacidade, aliada a uma série de desafios técnico/gerenciais) (Yoo, 2009).
\end{abstract}

No caso da radiodifusão, uma das maiores preocupações era a regulação do conteúdo, pensada para promover conteúdos desejáveis, do ponto de vista cultural e político, e programação com enfoque educativo (Yoo, 2009). Era comum, portanto, considerar-se a dissociação tecnológica de comunicação por voz, unidirecional, via cabos telefônicos, da programação de som e imagem, transmitida pelo espectro eletromagnético de radiodifusão.

De meados dos anos 1980 e a partir dos anos 1990, no entanto, o cenário no qual as telecomunicações se basearam durante décadas, começou a mudar por uma série de fatores, dos quais dois se destacam. O primeiro diz respeito à hegemonia no campo 
político/econômico de uma perspectiva neoliberal que, rompendo com o modelo de monopólio natural, geralmente sob controle do Estado, passa a privilegiar a competição nos diversos setores (a exemplo do desmembramento da AT\&T, da flexibilização das regras de concentração na TV americana, da privatização do setor de telefonia no Brasil e em dezenas de outros países, em todo o mundo). $\mathrm{O}$ segundo diz respeito à evolução das tecnologias de informação e comunicação, notadamente a intensificação do processo de digitalização, que cria as bases para um cenário de mídias convergentes.

$\mathrm{Na}$ verdade, com o advento do processo de digitalização, as diversas formas de comunicação passam a basear-se em códigos binários, o que possibilita que compartilhem uma mesma plataforma. Imagens, filmes, sons e palavras, convertidos para o formato digital, tiveram um significativo ganho no que diz respeito à velocidade, flexibilização e completude de comunicação. Isto possibilitou às malhas de info-telecomunicações a assimilação integral de produtos culturais, criando uma dinâmica de progressiva comercialização da cultura e da comunicação, o que alterou a forma de os sujeitos se relacionarem com os produtos culturais. Essas novas possibilidades técnicas, ao mesmo tempo em que geram projeções cumulativas formidáveis, bastante exploradas por empresas concessionárias de serviços de comunicação - em associação ou não com empresas da indústria cultural - têm atropelado os marcos regulatórios nacionais e internacionais (Smith, 1991).

À medida que o processo de digitalização se acelera, fica evidente que as diversas mídias passam a falar uma linguagem comum e, portanto, intercomunicativa, tanto do ponto de vista tecnológico, quanto de modelo de negócios. Assim, a partir dos anos 2000, intensificou-se o processo de convergência de mídias em plataformas tecnológicas, em organizações empresariais e nos modelos de 
negócio. A convergência tecnológica fornece a base que permite a apropriação e recirculação de conteúdos de toda natureza: num só momento uma pessoa pode expor-se a vários meios (telefone, TV, Internet), assim como pode criar conteúdos em múltiplos canais. Mas a viabilização disso depende totalmente de a exploração das tecnologias se organizar, de modo convergente, em função da oferta de serviços de comunicação e informação que, por sua vez, são igualmente convergentes.

Se as novas tecnologias de informação e comunicação criam desafios e oportunidades para as indústrias de mídia, também o fazem para seus usuários, crescentemente envolvidos numa cultura participativa intensa e, do ponto de vista do seu alcance geográfico e social, graças à convergência, cada vez mais extensa. Isto é, ao invés de consumidores passivos de mídia e serviços de informação e comunicação, temos agora utilizadores ativos e socialmente conectados, não mais silenciosos leitores/espectadores/ouvintes, mas ruidosos ativistas e publicistas.

O fenômeno da convergência envolve, pois, uma combinação de transformações interligadas e interdependentes, de natureza tecnológica, industrial/comercial, cultural e social, que afetam a regulação das comunicações. Compreender este fenômeno é essencial para vislumbrar um marco regulatório a ele apropriado.

Este artigo procura definir convergência e discutir suas conseqüências, tendo no horizonte o provável debate que se espera ocorra que no Brasil, ainda este ano, sobre a revisão do marco regulatório das comunicações.

\section{Convergência: delimitações do termo}

De uma maneira simples, a convergência, na sua vertente tecnológica, pode ser entendida como a fusão entre diversas 
plataformas e indústrias de mídia. Esse é o caso, por exemplo, do celular, que hoje desempenha atribuições que outrora eram típicas de um computador, na medida em que funde telefonia, computação e conteúdo. No entanto, o conceito deve ser entendido de forma mais ampla e não pode restringir-se apenas a questões de ordem tecnológica. Nas palavras de Jenkins:

[Convergence is] A word that describes technological, industrial, cultural, and social changes in the ways media circulates within our culture. Some common ideas referenced by the term include the flow of content across multiple media platforms, the cooperation between multiple media industries, the search for new structures of media financing that fall at the interstices between old and new media, and the migratory behavior of media audiences who would go almost anywhere in search of the kind of entertainment experiences they want. Perhaps most broadly, media convergence refers to a situation in which multiple media systems coexist and where media content flows fluidly across them. Convergence is understood here as an ongoing process or series of intersections between different media systems, not a fixed relationship."1 (2006, p. 282)

Bauer, por sua vez, considera que embora não exista uma definição universalmente aceita para o termo convergência, ele se refere mais frequentemente "to a blurring of the boundaries and/or a reduction of the differences between firms or industries. This blurring can occur in varying degrees, ranging from a partial overlap to a full

\footnotetext{
${ }^{1}$ [Convergência] É uma palavra que descreve mudanças tecnológicas, industriais, culturais e sociais na maneira de circulação da mídia em nossa cultura. Algumas idéias comumente referidas no termo incluem o fluxo de conteúdo através de plataformas de mídia diversas, a cooperação entre múltiplas indústrias de mídia, a busca por novas estruturas de financiamento das mídias que passa pelas interseções entre velha e nova mídia, e pelo comportamento migratório das audiências midiáticas que podem ir quase a qualquer lugar na busca pelo tipo de experiência de entretenimento que buscam. Talvez, de uma forma mais abrangente, a convergência midiática refere-se a uma situação na qual múltiplos sistemas de mídia coexistem e onde o conteúdo midiático flui livremente através deles. Convergência é entendida aqui como um processo em andamento ou uma série de interseções entre diferentes sistemas midiáticos, não uma relação fixa. (tradução nossa)
} 
elimination of differences and thus a fusion of formerly separate sectors $^{2} " .(2005$, p. 62)

Citando Geradin (2001), Bauer indica a possibilidade de uma "loose convergence" e de uma "deep convergence", e provê dois exemplos de cada um desses tipos de convergência:

An example of partial convergence is powerline communications, in which electricity wires are used to configure a platform for communications. Should powerline communications succeed, electric utilities would overlap with communications service providers in part of their operations, but the two sectors would not fully integrate. An example of deep convergence (or fusion) would be the emergence of fully integrated multimedia service providers, supplying their customers via integrated broadband communications platforms ${ }^{3} .(2005$, p. 62$)$

Bauer informa que se utilizou de autores diversos (incluindo Bauer, Weijnen, Turk \& Herder (2003) para distinguir três tipos de convergência - tecnológica, de mercado e organizacional. Ele deliberadamente exclui desta classificação um outro tipo: a convergência regulatória).

A convergência tecnológica, segundo ele, "refers to developments affecting the technological basis of communications at the level of networks, applications and services. The three most important

\footnotetext{
2 "a uma mescla das barreiras e/ou redução das diferenças entre firmas ou indústrias. Essa mescla pode ocorrer em vários níveis, desde uma interseção parcial até a completa eliminação de diferenças e conseqüentemente a fusão de setores outrora separados”. (tradução nossa)

3“Um exemplo de convergência parcial é a das comunicações via rede elétrica, na qual fios elétricos são usados para configurar uma plataforma para comunicações. Caso a comunicação via rede elétrica tenha sucesso, empresas de eletricidade poderão sobrepor-se com provedores de serviços de comunicação em parte de suas operações, mas os dois setores não se tornariam totalmente integrados. Um exemplo de convergência profunda (ou fusão) seria o surgimento se provedores de serviços multimídia totalmente integrados, suprindo seus clientes via plataformas de comunicação de banda larga integradas.” (tradução nossa)
} 
trends are digitization, increased processor speed and the migration to higher transmission capacity ${ }^{4} . "(2005$, p. 62).

Para definir a convergência de mercado, ele usa Greenstein \& Khanna (1997), que fazem uma análise do impacto da convergência no que se refere a produtos substitutos e complementares. De acordo com esses autores, "convergence in substitutes" implies that products or services become more interchangeable from a user perspective"5 (2005, p. 64). Já "Convergence in complements" occurs if two or more products or services work better together than they used to"6 (Greenstein \& Khanna, 1997 apud Bauer, 2005). Bauer indica que no caso da convergência de produtos substitutos, a competição aumenta na medida em que eles aumentam as opções para os usuários; no caso de convergência em produtos complementares, a tendência é que a competição seja reduzida e a cooperação entre as organizações aumente (2005, p. 64).

A convergência organizacional é definida por Bauer como a integração de firmas com funções diferentes, numa única organização:

Organizational convergence can be defined as the integration of functions formerly accomplished in separate firms into one enterprise. It can take many forms. For example, the emergence of multi-media companies through vertical and horizontal expansion into adjacent markets could be considered one form of organizational convergence. Alliances, network firms as

\footnotetext{
4 "refere-se desenvolvimentos que afetam a base tecnológica das comunicações nos níveis das redes, aplicações e serviços. As três tendências mais importantes são digitalização, incremente na velocidade de processadores e migração para capacidades de transmissão mais altas”. (tradução nossa)a

5 "convergência em substitutos" implica que produtos ou serviços tornem-se intercambiáveis a partir de uma perspectiva do usuário.” (tradução nossa)

6 “convergência em complementares" ocorre se dois ou mais produtos ou serviços funcionam melhor em conjunto.” (tradução nossa)
} 
well as mergers and acquisitions, are other forms of organizational convergence ${ }^{7}(2005$, p. 64$)$.

Ele adverte, contudo, que [....] "convergence is only one trajectory of the evolution of advanced communication systems. It is accompanied by parallel processes of differentiation and divergence" (2005, p. 65). Ele sintetiza seu raciocínio acerca do processo de convergência afirmando que:

Convergence is a reality, but it has shortcomings as an organizing lens to strategize and theorize the present and future of communication industries. Early views of convergence anticipated an amalgamation of the formerly separate voice, data, audio and video segments into one industry. Such forecasts tacitly, but erroneously assumed that technological convergence propelled by digitization, higher processing power of computers and higher bandwidth, would inevitably lead to market and organizational convergence. Whereas such developments do occur, they unfold in more multifaceted ways than commonly expected. Moreover, the effects of convergence on competition are not unanimously positive, as earlier analyses seemed to suggest. Other things equal, convergence in substitutes will intensify competition, but convergence in complements are likely to reduce it. As both processes unfold in parallel, it is difficult to predict the overall effect of convergence. Moreover, the economics of advanced communication networks and applications work against full convergence. Most importantly, firms facing high upfront and low incremental costs will have to differentiate their technology platforms, applications and services to be able to price discriminate and recover the initial investment. ${ }^{9}(2005$, p. 79$)$.

\footnotetext{
7“A convergência organizacional pode ser definida como a integração de funções que eram anteriormente desempenhadas por firmas distintas em uma única empresa. Ela pode tomar formas diversas. Por exemplo, o surgimento de empresas multimídia via expansão vertical e horizontal para mercados adjacentes pode ser considerada uma forma de convergência organizacional. Alianças, redes de firmas assim como fusões e aquisições, são outras formas de convergência organizacional.” (tradução nossa)

8"convergência é apenas uma trajetória da evolução dos sistemas de comunicação avançados. Ela é acompanhada por processos paralelos de diferenciação e divergências.” (tradução nossa)

9 "A convergência é uma realidade, mas tem as suas falhas como um meio para criar estratégias e teorizar o presente e o futuro das indústrias de comunicação. Visões iniciais acerca da convergência anteciparão uma fusão dos antes separados segmentos de voz, dados, áudio e vídeo numa única indústria. Tacitamente, mas
} 
E, finalmente, ressalta que há forte evidência de que o mercado se bifurcou; "on the one hand, convergence does integrate the technological basis and allows more integrated offerings; at the same time, the availability of service and pricing packages is highly fragmented and differentiated"10. $(2005$, p. 80)

Do ponto de vista econômico, a convergência se dá principalmente pela integração, sob domínio de um conglomerado empresarial, de empresas produtoras de bens simbólicos e materiais vinculados ao setor de informação e comunicações: televisão, provedores de conteúdo e de acesso à Internet, provedores de serviços de telecomunicações, jornais, revistas, produtoras de fonogramas, filmes e vídeos, editoras, produtores de jogos virtuais, entre outros.

Tão aclamada nos tempos atuais, a convergência não está, pois, desvinculada da práxis capitalista e se revela como uma oportunidade de maximização da acumulação de capital.

Em termos conceituais, a convergência se define como sendo a homogeneização dos suportes, produtos, lógicas de emissão e consumo das indústrias info-comunicacionais. Inicialmente tecnológico, esse processo, também chamado "revolução digital", supõe impactos em cenários relacionados com as culturas de

erroneamente, tais previsões assumiram que a convergência tecnológica, impulsionada pela digitalização, aumento da capacidade de processamentos de computadores e larguras de banda mais elevadas, iriam inevitavelmente levar à convergência de mercado e organizacional. Ainda que esses desenvolvimentos de fato aconteçam, eles se desdobram de forma mais multifacetadas do que o esperado. Além do mais, os efeitos da convergência em termos de competição não são totalmente positivos, como análises inicias pareciam sugerir. Em igualdade de condições, convergência em substitutos irá intensificar a competição, mas convergência em complementos irá possivelmente reduzi-la. Como os dois processos ocorrem em paralelo, é difícil prever o efeito final da convergência. Além do mais, os aspectos econômicos das redes e aplicações de comunicação avançadas trabalham contra a convergência completa. O elemento mais importante, é que firmas com custos iniciais altos e custos incrementais baixos terão de diferenciar suas plataformas, aplicações e serviços para serem capazes de diferenciar preços e recuperar seus investimentos iniciais”. (tradução nossa)

10 "por um lado, a convergência integra a base tecnológica e permite ofertas mais integradas; ao mesmo tempo, a disponibilidade de serviços e pacotes de preço é altamente fragmentada e diferenciada”. (tradução nossa) 
produção, as formas de organização, as rotinas de trabalho, os circuitos de distribuição e as lógicas de consumo de bens e serviços info-comunicacionais. Impulsionada pelos processos de globalização capitalista, está ancorada na incessante busca da velocidade, ou seja, a frenética mobilidade dos capitais (Miguel, 2010, p. 50).

Nessa linha mais crítica, Mosco and McKercher afirmam que a convergência é também um mito:

Convergence is not just a technological, political, and organizational process. It is also a myth or a story about how computer communication is revolutionizing technology, politics, and society. As such it is part of a sublime vision that, in its strongest form, envisions technology creating the conditions for the end of history, the end of geography, and the end of politics (Mosco, 2004). Convergence is therefore more than just a term to describe an ostensible change in technology and organization. It is part of a utopian discourse that aims to lead us from the coarse materiality of, in Nicholas Negroponte's terms, "the world of atoms," so that we can "learn to be digital" (Negroponte, 1996). This affirmative vision is used to rationalize deepening social inequalities, tightening surveillance practices, and the growing control of a handful of companies over the production and distribution of communication and information. To say that convergence is a myth is not to imply that it is false. Rather, myths take a basic empirical reality and enlarge it by attributing transformative social and cultural consequences that are not currently justified by empirical evidence ${ }^{11}$ (2006, p. 735)

\footnotetext{
11 “A convergência não é apenas um processo tecnológico, político e organizacional. É também um mito ou estória sobre como a comunicação via computadores está revolucionando a tecnologia, a política e a sociedade. Como tal, ela é parte de uma visão sublime, que na sua forma mais forte, antevê a tecnologia criando as condições para o fim da história, da geografia e da política (Mosco, 2004). A convergência é portanto mais do que um termo para descrever uma ostensiva mudança tecnológica e organizacional. É parte de um discurso utópico que busca nos conduzir da áspera materialidade do, nos termos de Nicholas Negroponte, “mundo de átomos”, para que possamos "aprender a ser digitais" (Negroponte, 1996). Essa visão afirmativa é usada para racionalizar desigualdades sociais profundas, fortalecer práticas de vigilância e o crescente controle de um punhado de empresas sobre a produção e distribuição de comunicação e informação. Dizer que a convergência é um mito não é simplesmente insinuar que ela é falsa. Pelo contrário, mitos tomam uma realidade empírica básica e a ampliam pela atribuição de conseqüências sociais e culturais
} 
A busca por novas possibilidades de mercado estaria, portanto, bastante atrelada ao desenvolvimento cada vez maior de tecnologias convergentes. Por conta disso, os avanços são dinâmicos, a criação de novos dispositivos é constante, interferindo diretamente na produção e no consumo de bens culturais. Segundo o raciocínio de Dantas (2009), a convergência tecnológica deve ser compreendida em sua relação com a horizontalização da cadeia produtiva da indústria cultural.

Definimos esse fenômeno que costuma ser denominado "convergência tecnológica" como um processo econômico, político e cultural que está fazendo convergir para um mesmo regime de negócios e de práticas sociais, o conjunto da cadeia produtiva da indústria cultural suportada em meios eletroeletrônicos de comunicação. Onde, até algumas poucas décadas atrás, tínhamos cadeias produtivas claramente diferenciadas e verticalizadas em função de seus negócios, práticas sociais e tecnologias apropriadas, tendemos a ter, de uns anos para cá e cada vez mais daqui para a frente, uma única cadeia horizontalizada, indiferente às distintas plataformas de comunicação ou transporte, mas segmentada conforme a divisão de trabalho, ou de valor, ao longo de todo o processo de produção, distribuição ou recepção de bens e serviços culturais mediatizados. Implica reconhecer que o processo não é determinado pela tecnologia conforme a expressão que se vai consagrando tende a induzir, mas resulta das contradições socioeconômicas que determinam a modelagem e evolução das tecnologias mesmas (DANTAS, 2009)

A horizontalização da cadeia produtiva se dá entre produtores, programadores e distribuidores de conteúdo, tendo como espinha dorsal a indústria fabricante de equipamentos e sistemas presentes em todas as etapas. As funções passam a ser compartilhadas de forma que a antiga distinção entre telecomunicações e radiodifusão deve ser desconsiderada em nome de operações convergentes de comunicação que oferecem seus pacotes de serviços e possibilidades (Dantas, 2009).

transformativas que não são correntemente justificáveis por dados empíricos”. (tradução nossa) 
Henten, Samarajiva e Melody (2003) consideram que indústrias que realizam integração vertical podem experimentar novas linhas de divisões de trabalho entre diferentes atores no campo. Cada uma das distintas indústrias pode ser concebida englobando os três níveis. A Tabela 1 procura demonstrar que existem muitas possibilidades de convergência em nível vertical, entre diferentes indústrias, e também integrando diferentes níveis. Convergência/integração e divergência/desintegração caminham lado a lado.

\begin{tabular}{|c|c|c|c|c|}
\hline \multicolumn{5}{|c|}{ Tabela 1 - Convergência/integração e divergência/desintegração } \\
\hline & $T I$ & Telecom & Radiodifusão & Outros meios \\
\hline $\begin{array}{l}\text { Conteúdo/ } \\
\text { Serviços }\end{array}$ & $\begin{array}{l}\text { Conteúdo } \\
\text { baseado } \\
\text { em } \\
\text { software }\end{array}$ & $\begin{array}{l}\text { Conteúdo baseado } \\
\text { em serviços de } \\
\text { telecomunicações }\end{array}$ & $\begin{array}{l}\text { Programas } \\
\text { de } \\
\text { radiodifusão }\end{array}$ & $\begin{array}{l}\text { Filmes, } \\
\text { músicas, } \\
\text { jornais, etc. }\end{array}$ \\
\hline $\begin{array}{l}\text { Transporte/ } \\
\text { software }\end{array}$ & software & Serviços de rede & Transmissão & $\begin{array}{l}\text { Cinemas, } \\
\text { videolocado } \\
\text { ras, etc. }\end{array}$ \\
\hline $\begin{array}{l}\text { Equipamentos } \\
\text { / Hardware }\end{array}$ & $\begin{array}{l}\text { Hardware } \\
\text { de TI }\end{array}$ & $\begin{array}{l}\text { Equipamento de } \\
\text { Telecom }\end{array}$ & $\begin{array}{l}\text { Equipament } \\
\text { o de } \\
\text { broadcastin } \\
\mathrm{g}\end{array}$ & $\begin{array}{l}\text { Reprodução } \\
\text { de filmes, } \\
\text { impressão, } \\
\text { etc. }\end{array}$ \\
\hline
\end{tabular}

Fonte: Samarajiva; Melody (2003 p. 28) - tradução nossa)

O nível horizontal prioritariamente se refere à convergência dos níveis de equipamento/ hardware e transporte/ software. Inclui também as possíveis implicações de convergência na camada de conteúdo, pois tipos de conteúdo que formalmente são dedicados a indústrias específicas, relativizam sua especificidade ao se converterem à plataforma digital. Isto representa novas possibilidades para usuários finais e novas possibilidades de mercado para produtores, ao mesmo tempo em que traz exigências de adaptações regulatórias (Henten, Samarajiva \& Melody, 2003, p. 27). 
Existe ainda um outro tipo de nível vertical, diferente daquele em que as empresas integram equipamento, transporte e conteúdo: é aquele em que países integram em sua regulação a infra-estrutura e o conteúdo. Exemplos disso são a Índia, o Reino Unido, Singapura e Malásia. Já na União Européia existe uma barreira rígida entre infraestrutura e conteúdo.

No caso da convergência horizontal há três níveis distintos, cada um associado a uma dimensão técnica: 1) a convergência na produção de conteúdo é relacionada primariamente à convergência de serviços; 2) a convergência em distribuição é relacionada à rede de convergência; 3) a convergência em produção de equipamento é relacionada ao terminal de convergência (Henten, Samarajiva e Melody, 2003, p. 28). Isso não significa apenas que diferentes plataformas podem ser usadas para prover os mesmos serviços, embora certa especialização seja mantida. Significa também que a formação de barreiras entre TI, telecomunicações, radiodifusão e outras empresas de mídia tornam-se menos visíveis e por conta disso deve-se ter em mente que para se tornar competitivo um conteúdo deve considerar as limitações e os potenciais de cada plataforma.

Com a digitalização as diferentes plataformas tornam-se cada vez mais intercambiáveis e adaptáveis umas às outras. O símbolo maior dessa sinergia é a internet, dado o seu caráter essencialmente híbrido. No entanto, outro meio merece destaque neste processo: o chamado celular, ou telefone móvel, que se converteu numa central de mídia digital (recebendo sinal de TV, acessando internet, armazenando arquivos, auxiliando a produção de conteúdo, etc.) e que tem tido notável influência na reestruturação do modelo de negócio que está se consolidando no setor.

A relativização de barreiras entre os meios é causa direta da interpenetração dos mercados de empresas concessionárias de 
serviços de telecomunicações e empresas concessionárias de radiodifusão.

Operadores de radiodifusão, em particular, estão urgentemente à procura de uma resposta para a pergunta como serviços interativos podem ser ativados. Estes serviços interativos são reconhecidos como os serviços de valor agregado para redes de transmissão que vai garantir a lucratividade no longo prazo. A combinação dos sistemas de transmissão com um canal de retorno via telefonia móvel poderia permitir novos modelos de serviços, especialmente se a mobilidade também é levada em conta no sistema de transmissão (Keller et. al., 2004, p. 279).

Enquanto radiodifusores buscam encontrar novas formas de distribuição de suas produções, empresários de telecomunicações investem na produção de conteúdo. No mercado brasileiro isso ocorre sem que haja qualquer ordenamento legislativo. 0 acirramento de disputas entre os concessionários de radiodifusão e os de serviços de telecomunicações - que envolvem fortes interesses políticos e econômicos - tem reflexos diretos nas esferas de decisão regulatória.

Braman (2004) afirma, ao tratar do caso americano, que definir o que seria uma política de mídia (podemos dizer comunicação) já foi fácil, mas hoje é muito complexo. Segundo ela, "When the Constitution and Bill of Rights were written, what we now call 'media policy' ensured that citizens were able to use oral and print means of expression to communicate with each other and with their government about the constitution of society ${ }^{12}$. (p. 155).

Agora a situação é bem mais complicada. Ela observa que "technological innovation created truly mass media, expanding the set of regulatory subjects and adding issues raised by interactions

\footnotetext{
12 “Quando a Constituição e a Lei de Direitos foram escritas, o que agora nós chamamos de 'política de mídia’ buscava assegurar que os cidadãos fossem capazes de fazer uso de meios de expressão oral e impressa para comunicarem-se uns com os outros e com seu governo acerca da formação da sociedade”. (tradução nossa)
} 
among media ${ }^{13}$." (Braman, 2004, p. 156). E o contexto tornou-se ainda mais complicado a partir do começo do século XXI:

By the opening of the twenty-first century, the field within which media policy operates has broadened yet again. Innovation has further transformed the fundamental nature of the technologies involved and the extent to which society is reliant upon those technologies. The directly communicative functions of the media are now a relatively small proportion of the overall role of information technologies in society. The distinction between public and private communicative contexts has become one of choice and will, rather than ownership, control and history of use. And we have come to understand that both non-political content and the infrastructure that carries it can have structural, or constitutive, impact ${ }^{14}$. (2004, p. 156)

Este novo cenário, segundo ela, comporta duas perspectivas distintas, ambas complexas individualmente e na sua inter-relação: "media policy" e "information policy". Neste sentido, ela adverte que

\begin{abstract}
"The phrase "the convergence of technologies" conflates several analytical issues pertinent to the problem of bounding the domain of media policy. New information technologies are qualitatively different from those with which media policy has historically dealt; blur medium, genre, function and industry; are ubiquitously embedded in the objects of the material world; and replace slow-changing structuration processes with more rapid processes best described as "flexible"15 (2004, p. 157).
\end{abstract}

\footnotetext{
13 “a inovação tecnológica criou mídias verdadeiramente de massa, o que expandiu o conjunto de temas sujeitos a regulação e adicionou questões ligadas à interação entre as mídias". (tradução nossa)

14 "No despontar do século vinte-e-um, o campo no qual a política de mídia opera se ampliou novamente. A inovação transformou a natureza fundamental das tecnologias envolvidas numa extensão na qual a sociedade depende dessas tecnologias. As funções diretamente comunicativas da mídia são agora uma parte relativamente pequena do papel das tecnologias de informação e comunicação na sociedade. A distinção entre contextos comunicativos públicos e privados tornou-se uma questão de escolha e vontade, ao invés de propriedade, controle e história de uso. E nós chegamos ao entendimento de que tanto conteúdos não-políticos quanto as infra-estruturas que os transmitem, podem ter impactos estruturais e constitutivos”. (tradução nossa)

15 "A frase 'convergência de tecnologias' une diversas questões analíticas pertinentes ao problema de 'amarrar' o campo de políticas de mídia. Novas tecnologias de informação são qualitativamente diferentes daquelas com as quais as
} 
Além disso, ela afirma que os suportes de mídia eram, no passado, distinguidos uns dos outros pelo número de receptores de mensagens (um, alguns ou muitos); pela natureza da interatividade, caso houvesse, entre emissor e receptor; e pela diferença entre sincronia e assincronia (2004, p.158). Ocorre que, segundo ela entende, a Internet funde tudo isto em todas as dimensões, criando a necessidade de dar conta dessa interação nas políticas de mídia. (idem, ibidem, p. 158-159).

A conseqüência, na visão de Braman, é uma mistura de meio, função e indústrias:

\begin{abstract}
"[...] confounds efforts to apply law and regulation that are industry-specific as well as efforts to use law and regulation (largely but not exclusively via antitrust law) to keep industries separate from each other. It disrupts habits of policy analysis because typically such techniques are based on assumptions about the social functions to be served by particular media industries. And it alters the economics of each of the industries involved, further disturbing habitual analytical assumptions ${ }^{16} . "(2004$, p. 159)
\end{abstract}

Face a tal dinamismo conceitual e contextual, decorrente do incessante desenvolvimento tecnológico e da tendência à convergência dos modelos de negócio, cabe questionar se o Estado é capaz de regular adequadamente a provisão de tais serviços. Segundo Kekic Laza,

"A maior parte dos Estados do mundo está mal equipada
para enfrentar o desafio (de participar ativamente na

políticas de mídia tem historicamente lidado; meios, gêneros, funções e indústrias fundem-se, e estão ubiquamente inseridos nos objetos do mundo material; e a substituição de mudanças lentas de estruturação de processos por processo mais rápidos, que são melhor descritos como flexíveis”. (tradução nossa)

${ }^{16}$ [....] dificulta os esforços para aplicar leis e regulações que são específicas para uma indústria assim como esforços no uso de leis e regulações (principalmente, mas não exclusivamente, via leis antitruste) para manter indústrias separadas umas das outras. Ela quebra hábitos de análise política por que, tipicamente, tais técnicas são baseadas em pressupostos acerca das funções sociais atendidas por indústrias de mídia específicas. E isso altera a lógica econômica de cada uma das indústrias envolvida, intensificando o distúrbio nos pressupostos analíticos habituais”. (tradução nossa) 
organização da sociedade). Dos 163 países incluídos num índice de capacidade do Estado elaborado pela Economist Intelligence Unit (EIU), apenas 34 são classificados como altamente capazes. A capacidade do Estado é considerada como moderada em 38 países. A maioria das nações tem Estados fracos (33) ou muito $\operatorname{fracos}^{17}(2011$, p. 94)

Há, além do mais, a questão da ideologia liberal, presente hoje mesmo nas formulações de teor socializante, que passaram a incluir conceitos tipicamente liberais, como Estado de direito, democracia representativa, economia de mercado, atração de capitais estrangeiros ${ }^{18}$. Esta ideologia fornece a base conceitual que conduz o Estado ao desinteresse por intervenção em atividades que de alguma forma se estruturem como atividade econômica. E certamente este é o caso das comunicações, sobretudo a partir dos anos 1980, quando começou a despontar a convergência tecnológica, econômica e organizacional, tanto na montagem da infra-estrutura quanto na prestação de serviços.

Uma perspectiva regulatória das comunicações deve, portanto, ser construída a partir das tendências para as quais apontam os desenvolvimentos presentes.

\footnotetext{
${ }^{17} \mathrm{O}$ Brasil está entre os 38 países com capacidade moderada. Laza esclarece que "(...) O índice da EIU baseia-se em uma avaliação (não ponderada) de 12 indicadores que coletivamente captam a capacidade de um Estado para exercer suas funções essenciais. Eles são: sua idade, sua história recente de estabilidade, a segurança de seus cidadãos, o nível de corrupção, o tamanho da economia paralela, o Estado de Direito, a qualidade de sua burocracia, se o governo controla todo o seu território, a extensão da influência estrangeira e o grau de fragmentação étnica, coesão social e confiança nas instituições públicas."

${ }^{18}$ Exemplo significativo disso foi a mudança de posição do Fórum Nacional de Democratização da Comunicação quanto à natureza das organizações que devem explorar serviços de comunicação. Em 1987, durante a Constituinte, o Fórum (então chamado Frente Nacional pela Democratização da Comunicação) propunha que todos os meios de comunicação fossem explorados por fundações de direito público. Nos anos 1993 a 1995 o Fórum negociou a chamada Lei do Cabo diretamente com os empresários do setor, defendendo que os serviços de cabodifusão deveriam ser explorados exclusivamente por empresas privadas.
} 


\section{Conclusão}

As exigências por reformulação que ecoam no mercado brasileiro de comunicações, em boa parte fundadas na convergência, fazem, portanto, parte de um processo mundial que pressiona os estados nacionais a re-conceituarem seus marcos legislativos. O problema está em que é essencial para esses estados, ao mesmo tempo em que evitem o atraso e garantam um avanço regulatório, assegurar a ampliação dos espaços virtuais nos quais seus cidadãos cada vez mais se realizam social, cultural, econômica e politicamente. Este objetivo pode conflitar - sobretudo na ausência de sólidos regulamentos com a exploração econômica dos serviços de informação e comunicação, mesmo quando se realize via empresas estatais. $\mathrm{Na}$ democracia, a solução está na transparência e no respeito à pluralidade e diversidade de visões existentes na sociedade.

A cobrança direcionada ao novo governo brasileiro, no que se refere à reformulação legislativa para o setor de comunicações, é grande. Ele ficou com a responsabilidade de tentar definir o interesse público e conciliá-lo, de um lado com os interesses dos radiodifusores, empresários de telecomunicações, investidores internacionais e, do outro, com as organizações da sociedade civil que participaram da Conferencia Nacional de Comunicação, patrocinada pelo próprio Governo.

Terá, portanto, que operar politicamente desde o seu interior - onde coexistem distintas tendências de compreensão e de interesses no setor - passando pelas representações da sociedade civil, aí incluídas as organizações empresariais e as chamadas organizações sociais até o Congresso Nacional, onde pululam e se interpenetram os mais variados interesses na regulamentação (ou não regulamentação) das comunicações. 
Em qualquer hipótese, qualquer que seja o caminho da conciliação escolhido pelo governo, parece tão evidente o alto grau de centralidade da convergência, nos termos aqui examinados, que dificilmente deixarão de prevalecer propostas que a considerem. Não que se possa prever domínio conceitual absoluto da convergência improvável, dada a existência de divergências múltiplas intra e inter todos os atores do processo sobre a aceitação dela como um dado irrecusável da realidade. Mas é perfeitamente previsível que inevitavelmente será um dos eixos principais tanto nos debates e negociações quanto no resultado legislativo que se conseguir. 


\section{Referências bibliográficas}

BAUER, Johannes. Communications \& strategies, no. 60, 4th quarter 2005, p. 59.

BAUER J.M., WEIJNEN M.P.C., TURK A.L. \& HERDER P.M. : "Delineating the scope of convergence in infrastructures: new frontiers for competition", in. W. A. H. THISSEN \& P.M. HERDER (Eds), Critical infrastructures ${ }^{-}$state of the art in research and application, pp. 209 231, Boston/Dordrecht/London: Kluwer, 2003.

BRAMAN, Sandra. Where has media policy gone? Defining the field in the twentyfirst century. Communication Law and Policy, v. 9, number 2, Spring 2004

DANTAS, Marcos. Convergência digital: entre os "jardins murados" e as praças públicas. In: SEL, Susana (org.). Políticas de comunicacion el el capitalismo contemporâneo: América Latina y sus encrucijadas. Buenos Aires: Consejo Latinoamericano de Ciencias Sociales - CLACSO, 2010.

GREENSTEIN S. \& KHANNA T.: "What does industry convergence mean?" in: D.B. YOFFIE (Ed.), Competition in the age of digital convergence, pp. 201-226, Boston: Harvard Business School Press, 1997.

HENTEN, Anders; SAMARAJIVA, Rohan; MELODY, William. Designing next generation telecom regulation: ICT convergence or multi-sector utility? info, Vol. 5, 2003, pp 26-33.

JENKINS, Henry. Convergence Culture: where old and new media collide. New York: New York University Press, 2006.

KEKIC, Laza. O Estado do Estado. O mundo em 2011, São Paulo: Carta Capital, janeiro/fevereiro 2011, p. 94.

KELLER et. al. Convergence of Broadcast and New Telecom Networks. Wireless Personal Communications. N. 17: 269-282, 2001.

MIGUEL, João. Convergência tecnológica e implicações político-comunicacionais. In BRITTOS, Valério (org.). Digitalização, diversidade e cidadania: convergências Brasil e Moçambique. São Paulo: Annablume, 2010. 
MOSCO, Vincent; MCKERCHER, Catherine. (2006). Convergence Bites Back: Labour Struggles in the Canadian Communication Industry. Canadian Journal of Communication, Vol 31, No 3 (2006).

SMITH, Anthony. The Age of Behemoths - The Globalization of Mass Media Firms. New York: Priority Press Publications, 1991.

YOO, Christopher. The convergence of broadcasting and telephony: legal and regulatory implications. Communications \& convergence review. Vol 01, N.1, pp. 44-55, 2009.

COMMOLITICA
A Revista Compolítica é uma revista eletrônica da Associação Brasileira de Pesquisadores em Comunicação e Política. Com periodicidade semestral, sua proposta é difundir a produção acadêmica relacionada às interfaces desses campos de estudo.

Ao citar este artigo, utilize a seguinte referência bibliográfica

JAMBEIRO, Othon, FERREIRA, Fábio, BARROS e Chalini. LA Convergência como Condicionante da Regulação das Comunicações. In: Revista Compolítica, n. 1, vol. 1, ed. março-abril, ano 2011. Cidade: Compolítica, 2011. 


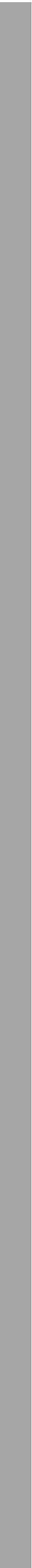

